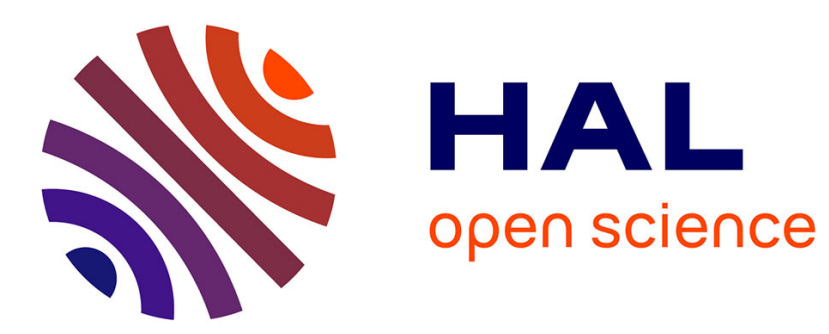

\title{
Geodesic flow of the averaged controlled Kepler equation
} Bernard Bonnard, Jean-Baptiste Caillau

\section{To cite this version:}

Bernard Bonnard, Jean-Baptiste Caillau. Geodesic flow of the averaged controlled Kepler equation. 2008. hal-00134702v6

\section{HAL Id: hal-00134702 https://hal.science/hal-00134702v6}

Preprint submitted on 22 Feb 2008

HAL is a multi-disciplinary open access archive for the deposit and dissemination of scientific research documents, whether they are published or not. The documents may come from teaching and research institutions in France or abroad, or from public or private research centers.
L'archive ouverte pluridisciplinaire HAL, est destinée au dépôt et à la diffusion de documents scientifiques de niveau recherche, publiés ou non, émanant des établissements d'enseignement et de recherche français ou étrangers, des laboratoires publics ou privés. 


\title{
Geodesic flow of the averaged controlled Kepler equation
}

\author{
Bernard Bonnard* Jean-Baptiste Caillau ${ }^{\dagger}$
}

\begin{abstract}
A normal form of the Riemannian metric arising when averaging the coplanar controlled Kepler equation is given. This metric is parameterized by two scalar invariants which encode its main properties. The restriction of the metric to $\mathbf{S}^{2}$ is shown to be conformal to the flat metric on an oblate ellipsoid of revolution, and the associated conjugate locus is observed to be a deformation of the standard astroid. Though not complete because of a singularity at the origin in the space of ellipses, the metric has convexity properties that are expressed in terms of the aforementioned invariants, and related to surjectivity of the exponential mapping. Optimality properties of geodesics of the averaged controlled Kepler system are finally obtained thanks to the computation of the cut locus of the restriction to the sphere.
\end{abstract}

Keywords. orbit transfer, Riemannian metrics, cut and conjugate loci

MSC classification. 49K15, 70Q05

\section{Introduction}

The mechanical system we are interested in is the Kepler equation with control appearing linearly in the right hand side,

$$
\ddot{q}=-q /|q|^{3}+u
$$

thus modelling the action of the engine of a spacecraft in a central field, e.g., the Earth gravitational field. In such a model, several approximations are made: Essentially, the spacecraft mass variation - which would reflect fuel consumptionis not taken into account, and higher order terms of the potential as well as influence of other celestial bodies are neglected. Fixing initial and terminal conditions, one defines an orbit transfer problem between Keplerian orbits [6]. Though the full model is three-dimensional, we restrict here the discussion to the coplanar case where the position, $q$, and the control, $u$, lie in $\mathbf{R}^{2}$. This is indeed sufficient to analyze orbit transfers with small change in the inclination

\footnotetext{
*Math. Institute (UMR CNRS 5584), Bourgogne University, 9 avenue Savary, F-21078 Dijon (bernard.bonnardQu-bourgogne.fr).

${ }^{\dagger}$ ENSEEIHT-IRIT (UMR CNRS 5505), Toulouse University, 2 rue Camichel, F-31071 Toulouse (caillau@n7.fr).
} 
(with respect to the equatorial plane for instance, in the case of Earth orbits). The criterion which is physically important is the minimization of the consumption. It amounts to minimizing the $\mathrm{L}^{1}$-norm of the control over a fixed period of time $\left(t_{f}\right.$ being the transfer time),

$$
\int_{0}^{t_{f}}|u| \mathrm{d} t \rightarrow \min .
$$

This performance index only makes sense provided a constraint on the control is added,

$$
|u| \leq \eta
$$

As before, the finite dimensional norm $|$.$| is a 2-norm, |u|=\sqrt{\left|u_{1}\right|^{2}+\left|u_{2}\right|^{2}}$. In modern space missions, low propulsion is considered, so $\eta$ is a first small parameter of the problem.

Using the mechanical energy of the system, $E=\dot{q}^{2} / 2-1 /|q|$, one defines the elliptic domain in the $(q, \dot{q})$-space,

$$
Q=\{E<0, q \wedge \dot{q} \neq 0\} .
$$

On this domain, new coordinates are available, namely those first integrals of the unperturbed motion that describe the geometry of the osculating orbit which is an ellipse when the energy is negative. Consider hence the semi-latus rectum of the ellipse, $P$, together with its eccentricty vector, $\left(e_{x}, e_{y}\right)$ : Adding the longitude, $l$, to define the position of the spacecraft on its orbit, the elliptic domain is clearly seen to be fibered over $\mathbf{S}^{1}$ since

$$
Q=\left\{P>0,\left|\left(e_{x}, e_{y}\right)\right|<1, l \in \mathbf{S}^{1}\right\},
$$

and the dynamics in these orbital elements takes the form of the so-called Gauss equations,

$$
\mathrm{d} x / \mathrm{d} l=u_{1} F_{1}(l, x)+u_{2} F_{2}(l, x), \quad i=\omega(l, x),
$$

where $x=\left(P, e_{x}, e_{y}\right)$, and where the $F_{i}$ are vector fields over the space of ellipses, $X_{e}=\left\{P>0,\left|\left(e_{x}, e_{y}\right)\right|<1\right\}$, periodically parameterized by longitude. More precisely,

$$
\begin{aligned}
F_{1}= & \left(P^{2} / W^{2}\right)\left(\sin l \partial / \partial e_{x}-\cos l \partial / \partial e_{y}\right), \\
F_{2}= & \left(P^{2} / W^{2}\right)[2 P / W \partial / \partial P+ \\
& \left.\left(\cos l+\left(e_{x}+\cos l\right) / W\right) \partial / \partial e_{x}+\left(\sin l+\left(e_{y}+\sin l\right) / W\right) \partial / \partial e_{y}\right],
\end{aligned}
$$

while

$$
\omega=W^{2} / P^{3 / 2}, \quad W=1+e_{x} \cos l+e_{y} \sin l .
$$

In the context of low propulsion, it is natural to consider orbit transfers with many revolutions, and the inverse of the final longitude (taken as the new independent variable), $\varepsilon=1 / l_{f}$, is the second small parameter of the system.

Analysis in the minimum time case [5] gives evidence for the existence of conjugate points where extremals of the system cease to be locally minimizing. A simplified approximation of the system is then desirable to study optimality properties. A relaxation of the problem consists in replacing the $\mathrm{L}^{1}$ criterion by an $\mathrm{L}^{2}$ one,

$$
\int_{0}^{t_{f}}|u|^{2} \mathrm{~d} t=\int_{0}^{l_{f}}|u|^{2} \mathrm{~d} l / \omega \rightarrow \min
$$


then in dropping the constraint on the control. Minimizing trajectories are projections of extremals parameterized by Pontryagin maximum principle. Such curves are integrals of the following Hamiltonian on the cotangent bundle $T^{*} X_{e}$,

$$
H(l, x, p)=(\omega / 2)\left(H_{1}^{2}+H_{2}^{2}\right)
$$

where the $H_{i}(l, x, p)=\left\langle p, F_{i}(l, x)\right\rangle$ are Hamiltonian lifts of the vector fields. As in [10], averaging with respect to longitude is introduced and $\mathscr{C}^{0}$-approximations of trajectories of $H(l, x, p)$ on intervals whose length is of order $1 / \varepsilon$ are provided by trajectories of

$$
H(x, p)=(2 \pi)^{-1} \int_{0}^{2 \pi} H(l, x, p) \mathrm{d} l .
$$

Fine numerical simulations [5] actually demonstrate that, for very low propulsion, only the averaged behaviour of the trajectories is observed. Computing and using an adapted set of orbital elements, $x=(n, e, \theta)$, where $n=[(1-$ $\left.\left.e^{2}\right) / P\right]^{3 / 2}$ is the mean motion and where $(e, \theta)$ are polar coordinates on the Poincaré disk, D-one gets 3 ]

$$
H=(1 / 2)\left[9 n^{1 / 3} p_{n}^{2}+5\left(1-e^{2}\right) p_{e}^{2} /\left(2 n^{5 / 3}\right)+\left(5-4 e^{2}\right) p_{\theta}^{2} /\left(2 n^{5 / 3} e^{2}\right)\right] .
$$

Since the averaged Hamiltonian is quadratic in the adjoint variable with full rank, extremals are interpretated as geodesics of the Riemannian metric

$$
g=d n^{2} /\left(9 n^{1 / 3}\right)+2 n^{5 / 3} d e^{2} /\left(5\left(1-e^{2}\right)\right)+2 n^{5 / 3} e^{2} d \theta^{2} /\left(5-4 e^{2}\right) .
$$

The singularities are $n=0$ and $e=1$, while $e=0$ is only due to the use of polar coordinates on $\mathbf{D}$ and removed by taking $\left(e_{x}, e_{y}\right)$ instead.

The first section of the paper is devoted to curvature computations. An analytic prolongation of the metric is considered, and a normal form is obtained, revealing two scalar invariants. The first one is related to convexity issues, whereas the second defines the geometry of the restriction of the metric to $\mathbf{S}^{2}$ and counts the number of closed simple geodesics on the sphere. We prove in the second section that the metric is integrable in the class of harmonic functions, and provide explicit quadratures in suited coordinates. An estimation of the length of closed geodesics combined with the curvature evaluation of $\$ 1$ allows to compute the injectivity radius of the metric restricted to $\mathbf{S}^{2}$ and to devise a necessary condition for optimality of metrics in the normal form derived. In Kepler's case, this condition is not fulfilled and cut points exist for the analytically extended metric. Section 3 deals with convexity issues. Though the singularity at the origin of the metric results in incompleteness, convexity may occur and is characterized thanks to the two previously mentioned parameters. The analysis is reduced to a discussion in two-dimensional meridian half-planes, and related to surjectivity of the exponential mapping. In the last section, we compute the cut locus of the metric restricted to the sphere and the astroid-like associated conjugate locus. Both are related to the separating line and conjugate locus of the full three-dimensional metric.

\section{Curvature of the system}

We begin with an analytic prolongation of the metric from the space of ellipses, $X_{e}=\mathbf{R}_{+}^{*} \times \mathbf{D}$, which is homeomorphic to the product of the positive real line 
with one open hemisphere, to $X=\mathbf{R}_{+}^{*} \times \mathbf{S}^{2}$, product of the positive real line with the full two-dimensional sphere.

Let $r=(2 / 5) n^{5 / 6}$, and let $(\theta, \varphi)$ be the usual angular coordinates on the two-sphere. We set $e=\sin \varphi$, thus lifting $\mathbf{D}$ onto $\mathbf{S}^{2}$, and get the following.

Proposition 1. A normal form of Kepler's metric (1) is

$$
g=\mathrm{d} r^{2}+\left(r^{2} / c^{2}\right)\left(G(\varphi) \mathrm{d} \theta^{2}+\mathrm{d} \varphi^{2}\right)
$$

which is analytic on $X=\mathbf{R}_{+}^{*} \times \mathbf{S}^{2}$ with

$$
G(\varphi)=\sin ^{2} \varphi /\left(1-\left(1-\mu^{2}\right) \sin ^{2} \varphi\right)
$$

and positive $c, \mu, \mu \leq 1$. In Kepler's case, $c=\sqrt{2 / 5}$ and $\mu=1 / \sqrt{5}$.

The singularities $\varphi=0(\pi)$ at the poles are simply due to the choice of coordinates on $\mathbf{S}^{2}$, and $r=0$ is the only singularity left since $e=1$ is absorbed as the equator, $\varphi=\pi / 2$.

The restriction of the metric to $\{r=c\} \simeq \mathbf{S}^{2}$ is $g_{2}=G(\varphi) \mathrm{d} \theta^{2}+\mathrm{d} \varphi^{2}$, and we denote similarly the resulting normal forms of Hamiltonians:

$$
H=p_{r}^{2} / 2+\left(c^{2} / r^{2}\right) H_{2}, \quad H_{2}=(1 / 2)\left(p_{\theta}^{2} / G(\varphi)+p_{\varphi}^{2}\right) .
$$

An analytic metric on the two-sphere of revolution 9, 14 like $g_{2}$ turns to be isometric to $f(z) g_{0}$, where $g_{0}=\sin ^{2} \varphi \mathrm{d} \theta^{2}+\mathrm{d} \varphi^{2}$ is the restriction of the flat metric to $\mathbf{S}^{2}$ and $f$ a positive function of the vertical coordinate $z$ (see [2]). A more specific construction is available here.

Proposition 2. The metric $g_{2}$ is conformal to the flat metric restricted to an oblate ellipsoid of revolution with unit semi-major axis and semi-minor axis $\mu$.

Proof. We have indeed

$$
g_{2}=G(\varphi) \mathrm{d} \theta^{2}+\mathrm{d} \varphi^{2}=g_{1} /\left(1-\left(1-\mu^{2}\right) \sin ^{2} \varphi\right),
$$

where $g_{1}=\sin ^{2} \varphi \mathrm{d} \theta^{2}+\left(1-\left(1-\mu^{2}\right) \sin ^{2} \varphi\right) \mathrm{d} \varphi^{2}$ is the restriction of the flat three-dimensional metric to the ellipsoid parameterized by

$$
x=\sin \varphi \cos \theta, \quad y=\sin \varphi \sin \theta, \quad z=\mu \cos \varphi,
$$

whence the result.

Accordingly, there is a natural homotopy from $g_{2}$ to the flat metric on $\mathbf{S}^{2}$, having the parameter $\mu$ varying up to $\mu=1$ (since $g_{2 \mid \mu=1}=g_{1_{\mid \mu=1}}=g_{0}$ ). This resemblance with the ellipsoid of revolution defined by $\mu$ is crucial to understand the cut and conjugate loci computations of $\$ 4$.

When $r \rightarrow \infty$, the semi-major axis coordinate $a=(5 r / 2)^{-4 / 5}$ tends to zero, the collision point in the space of ellipses. We analyse the effect of the collision on the curvature.

Let $V$ be the span of $\partial / \partial \theta$ and $\partial / \partial \varphi$. The sectional curvature $K_{V}$ of $g$ is $R_{2323} /|\partial / \partial \theta \wedge \partial / \partial \varphi|^{2}$ where $R$ is the Riemannian curvature tensor and $R_{2323}=$ $R(\partial / \partial \theta, \partial / \partial \varphi, \partial / \partial \theta, \partial / \partial \varphi)$.

Lemma 1. The metric $g$ is flat if and only if $K_{V}$ is zero. 
Proof. The condition is clearly necessary. It is also sufficient since, because of the normal form of the metric, four out of five components of the curvature tensor are zero. The fifth is precisely the sectional curvature $K_{V}$.

Proposition 3. The sectional curvature of $g$ in Kepler's case is

$$
K_{V}=\left(1-24 \cos ^{2} \varphi-16 \cos ^{4} \varphi\right) /\left(r^{2}\left(1+4 \cos ^{2} \varphi\right)^{2}\right) \rightarrow 0, \quad r \rightarrow \infty,
$$

and the metric is asymptotically flattened by the collision.

We show in 4 that the analysis of optimality of the geodesic flow of $g$ is deduced from the properties of the restriction to $\mathbf{S}^{2}$. We immediately compute the Gauss curvature of $g_{2}, K=-\left(\mathrm{d}^{2} \sqrt{G} / \mathrm{d} \varphi^{2}\right) / \sqrt{G}$.

Proposition 4. The Gauss curvature of $g_{2}$ is

$$
K=\left(\mu^{2}-2\left(1-\mu^{2}\right) \cos ^{2} \varphi\right) /\left(1-\left(1-\mu^{2}\right) \sin ^{2} \varphi\right)^{2} .
$$

The curvature reaches its maximum, $K=1 / \mu^{2}$, along the equator.

By Rauch theorem, the first conjugate time along any geodesic on $\mathbf{S}^{2}$ is then bounded below by $\mu \pi$, and this bound is optimal since it is reached on the equator $\varphi=\pi / 2$ where the curvature is constant. In particular, the injectivity radius, $i\left(\mathbf{S}^{2}\right)$, which is the infimum of distances of points to their respective cut loci (see \$4), is known [8] to be reached - the manifold is compact - either at a conjugate point, or at the half of a simple closed geodesic. The task of computing periodic geodesics on the sphere is completed in next section, thus providing a first necessary condition for global optimality of $g$ thanks to the estimation of this injectivity radius.

\section{$2 \quad$ Integrability}

The unperturbed Kepler motion is classically integrable, and so remains the averaged controlled one, independently of the choice of parameters $c, \mu$ in (2).

Proposition 5. The coordinate $\theta$ is cylic, and $\mathrm{H}, \mathrm{H}_{2}, p_{\theta}$ are three independent first integrals in involution. On $\mathbf{S}^{2}$, the linear first integral $p_{\theta}$ verifies the Clairaut relation [2, 77], $p_{\theta}=\cos (\phi) \sqrt{G(\varphi)}$, where $\phi$ is the angle of the geodesic with a parallel.

The geodesic flow on $X=\mathbf{R}_{+}^{*} \times \mathbf{S}^{2}$ is thus Liouville-integrable, which we can also check by a direct computation of $r$ on the level set $H=1 / 2$ (parameterization of geodesics by arc length).

Lemma 2. The coordinate $r^{2}$ is a degree two polynomial depending only on $r_{0}$ and $p_{r_{0}}, r^{2}=t^{2}+2 r_{0} p_{r_{0}} t+r_{0}^{2}$.

The integration is then performed using the time change $d \tau=d t / c^{2}$, and one readily gets

$$
\tau\left(t, r_{0}, p_{r_{0}}\right)=c^{2}\left(\arctan \left(t /\left(r_{0} \cos \alpha_{0}\right)+\tan \alpha_{0}\right)-\alpha_{0}\right) /\left(r_{0} \cos \alpha_{0}\right)
$$

with $p_{r_{0}}=\sin \alpha_{0}$. Whenever $p_{r_{0}}= \pm 1$, the angles $\theta$ and $\varphi$ are constant on $H=1 / 2$, and we set $\tau=0$. Parameterizing again extremals by arc length 


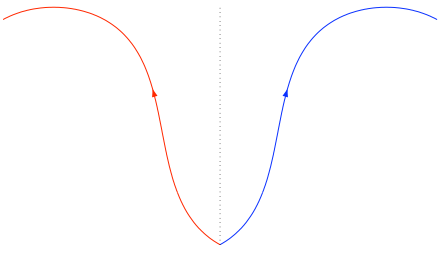

$s_{1}$

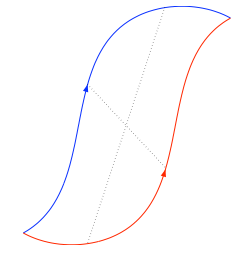

$s_{2}$

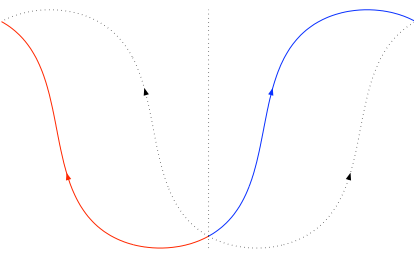

$s_{1} s_{2}$

Figure 1: Action of the Klein group on geodesics.

on $\{r=c\} \simeq \mathbf{S}^{2}$, we set $H_{2}=1 / 2$ and proceed to the integration of $g_{2}$, first underlying the symmetries of the system.

Since $\theta$ is cyclic, we can normalize $\theta_{0}$ to zero, and the action of the two reflections $s_{1}: p_{\theta} \mapsto-p_{\theta}, s_{2}: p_{\varphi} \mapsto-p_{\varphi}$ on the Hamiltonian $H_{2}$ is clear: $s_{1}$ defines an axial symmetry in the $(\theta, \varphi)$-plane with respect to $(O \varphi)$, while $s_{2}$ is a central symmetry $(\theta, \varphi) \mapsto\left(2 \theta_{c}-\theta, \pi-\varphi\right), \theta_{c}$ depending on the geodesic. These two reflections generate the Klein group, $\mathbf{V} \simeq \mathbf{Z} / 2 \mathbf{Z} \times \mathbf{Z} / 2 \mathbf{Z}$, which defines an Abelian discrete group of symmetries of $\mathrm{H}_{2}$, see Fig. 1

Proposition 6. Geodesics on $H_{2}=1 / 2$ are parameterized by $p_{\theta}$, and the coordinate $\varphi$ is $T$-periodic with $T=4 \pi / a$ and $a=2 \sqrt{1+\left(1-\mu^{2}\right) p_{\theta}^{2}}$. On a quarter of period $\left[t_{1}, t_{1}+T / 4\right]$, for $p_{\varphi_{0}}$ nonnegative,

$$
\begin{aligned}
\theta & =\operatorname{sign}\left(p_{\theta}\right)\left[\arctan \left(\tan \left(a\left(t-t_{1}\right) / 2\right) / \sqrt{b}\right)\right]_{0}^{t}-\left(1-\mu^{2}\right) p_{\theta} t \\
\varphi & =\arcsin \sqrt{(1+b) / 2-(1-b) \cos \left(a\left(t-t_{1}\right)\right) / 2}
\end{aligned}
$$

with $t_{1}=-\pi /(2 a)-\arcsin \left(\left(2 \sin ^{2} \varphi_{0}-1-b\right) /(1-b)\right) / a$ and $b=p_{\theta}^{2} /(1+(1-$ $\left.\left.\mu^{2}\right) p_{\theta}^{2}\right)$. The quadratures are extended by analyticity on the whole period, and obtained for negative $p_{\varphi_{0}}$ using a $-2 t_{1}$ time translation on $\varphi$ and $\dot{\theta}$,

$$
\varphi_{\mid p_{\varphi_{0}}<0}(t)=\varphi_{\mid-p_{\varphi_{0}}}\left(t+2 t_{1}\right), \quad \theta_{\mid p_{\varphi_{0}}<0}(t)=\theta_{\mid-p_{\varphi_{0}}}\left(t+2 t_{1}\right)-\theta_{\mid-p_{\varphi_{0}}}\left(2 t_{1}\right) .
$$

Remark. Inner symmetries on each geodesic imply that, for $p_{\varphi_{0}}$ nonnegative, $\varphi(t)=\pi-\varphi\left(2\left(t_{1}+T / 4\right)-t\right)$ on $\left[t_{1}+T / 4, t_{1}+T / 2\right]$, and $\varphi(t)=\varphi\left(2\left(t_{1}+T / 2\right)-t\right)$ on $\left[t_{1}+T / 2, t_{1}+T\right]$. Similar relations hold for $\theta$, since $\dot{\theta}=p_{\theta} / G(\varphi)$ is periodic.

Corollary 1. The metric $g$ is integrable in the class of harmonic functions.

When $p_{\varphi_{0}}=0$, we get the equator if $\varphi_{0}=\pi / 2$, the so-called pseudo-equators otherwise.

Corollary 2. All geodesics with the exception of meridians, $\theta=\mathrm{cst}$, are pseudoequators.

Proof. Excluding meridians, on $H_{2}=1 / 2$ one always has $0<p_{\theta}^{2} \leq G\left(\varphi_{0}\right) \leq$ $1 / \mu^{2}$, and there is $\varphi_{0}^{\prime}$ in $[0, \pi / 2]$ such that $p_{\theta}^{2}=G\left(\varphi_{0}^{\prime}\right)$. The geodesic is a pseudo-equator for the new initial condition $\varphi_{0}^{\prime}$.

According to Proposition $6, \dot{\theta}$ is periodic, and the variation of $\theta$ which is nondecreasing over a period (we restrict ourselves to $p_{\theta} \geq 0$ by symmetry) is

$$
\Delta \theta=2 \pi\left(1-\left(1-\mu^{2}\right) e_{0}\right)
$$


for a pseudo-equator of initial condition $e_{0}=\sin \varphi_{0}>0$. This expression is also valid for meridians passing through the solvable singularity $e_{0}=0$. Indeed, there are jumps in $\theta$ at the poles generating the whole family of meridians: $\theta(\varphi=0+)=\mathrm{cst}, \theta(\varphi=\pi)=\mathrm{cst}+\pi, \theta(\varphi=2 \pi)=2 \pi$, for a total variation $\Delta \theta=2 \pi$. As $\Delta \theta /(2 \pi) \leq 1$, closed geodesics - including meridians - are at least one period long. For pseudo-equators of initial condition $e_{0}$ in 10,1$]$ or meridians $\left(e_{0}=0\right), T=2 \pi \sqrt{1-\left(1-\mu^{2}\right) e_{0}^{2}}$ by Proposition 6 , so that the half-length of any closed geodesic is bounded below by $\mu \pi$.

Theorem 1. The injectivity radius of the metric $g$ restricted to $\{r=c\} \simeq \mathbf{S}^{2}$ is $i\left(\mathbf{S}^{2}\right)=\mu \pi$. A necessary condition for global optimality of $g$ on $X=\mathbf{R}_{+}^{*} \times \mathbf{S}^{2}$ is $c / \mu \leq 1$.

Proof. According to the discussion at the end of $\left\{1, i\left(\mathbf{S}^{2}\right) \leq \mu \pi\right.$, whence the equality given the lower bound of the half-length of closed geodesics. Let us show that $g$ is not globally optimal if $c>\mu$. The time $\sigma$ on the level set $H_{2}=1 / 2$ is given by $(3)$, up to a renormalization by $H_{2 \mid H=1}$, that is

$$
\sigma\left(t, r_{0}, p_{r_{0}}\right)=c\left(\arctan \left(t /\left(r_{0} \cos \alpha_{0}\right)+\tan \alpha_{0}\right)-\alpha_{0}\right) \text {. }
$$

Then

$$
\sup _{\left|p_{r_{0}}\right|<1} \sup _{t \geq 0} \sigma\left(t, r_{0}, p_{r_{0}}\right)=c \pi,
$$

this bound not being reached on any geodesic. Let now $\gamma$ be a geodesic of length $\mu \pi$ on $\mathbf{S}^{2}$ with one cut point. Since $\mu \pi<c \pi$, this geodesic is the projection on $\mathbf{S}^{2}$ of a geodesic on $X$ (see Lemma 4 in $\$ 4$ which cannot be globally minimizing because of the cut point on $\gamma$.

Remark. For the condition to be also sufficient, we would need the metric on $X$ to be complete so as to use the standard structure result on the cut locus (decomposed into conjugate points and points on the separating line, see 4 . This is not the case, as will be stated in $\$ 3$ The limit case is the flat case, $c=\mu=1$ and $X \simeq \mathbf{R}^{3}-\{0\}$ where global optimality holds.

Corollary 3. The metric is not globally optimal on $X$ in Kepler's case $(c / \mu=$ $\sqrt{2}>1)$.

As $\dot{\theta}$ and $\varphi$ share the same period, closed geodesics on $\mathbf{S}^{2}$ are characterized by the fact that $\Delta \theta /(2 \pi)$ be rational (except for the equator, $e_{0}=1$, which is the only geodesic with constant $\varphi$ ). Conversely, a geodesic which is a pseudo-equator for $e_{0}$ such that the quotient is irrational densely fills the strip $\left[\varphi_{0}, \pi-\varphi_{0}\right]$ on the sphere. In Kepler's case where $\mu^{2}$ is rational, there is one closed geodesic passing through every rational $e_{0}$ since $\Delta \theta /(2 \pi)=1-\left(1-\mu^{2}\right) e_{0}$. It is known 13 that there exist at least three simple closed geodesics, and there are actually infinitely many of them - the meridians - because of the symmetry of revolution. We thus discuss the existence of simple closed geodesics modulo rotations on $\theta$.

Proposition 7. There are exactly $\left[1 / \mu^{2}\right]$ simple closed geodesics modulo rotations around the poles on $\left(\mathbf{S}^{2}, g_{2}\right)$.

Remark. The invariant $\mu$ thus measures the number of simple closed geodesics on the sphere. The result degenerates for $\mu=1$, all great circles being meridians for appropriate axes on the flat two-sphere. 
Proof. For closed geodesics, $\Delta \theta /(2 \pi)$ is rational,

$$
1-\left(1-\mu^{2}\right) e_{0}=p / q,
$$

and simple ones are obtained for $p=1$. Then $e_{0}=(q-p) /\left(q\left(1-\mu^{2}\right)\right)_{\mid p=1} \leq 1$, so $1 \leq q \leq 1 / \mu^{2}$.

In Kepler's case, $1 / \mu^{2}=5$ and there are five classes of simple closed geodesics (see Fig. 2) among which meridians and pseudo-equator for $e_{0}=5 / 6$ have $\pi$ rational lengths $(2 \pi$ and $4 \pi$, respectively). In fact, it appears that the existence of closed geodesics with length in $\pi \mathbf{Q}$ is expressed in terms of a standard Diophantine equation, the Pell equation [16. When $\mu^{2}$ is indeed rational, such closed geodesics are obtained finding a rational $e_{0}$ such that $T /(2 \pi)=$ $\sqrt{1-\left(1-\mu^{2}\right) e_{0}^{2}}$ belongs to $\mathbf{Q}$, that is solving the quadratic Diophantine equation

$$
a^{2}-\left(1-\mu^{2}\right) b^{2}=c^{2} .
$$

This equation is a generalized Pell equation, parameterized by $c$. In Kepler's case, 9 takes the form $5 a^{2}-4 b^{2}=5 c^{2}$ (with $e_{0}=b / a$ ), which is reduced to the standard equation

$$
x^{2}-D y^{2}=25 c^{2},
$$

where $D=20$ is not a perfect square, and where $a=x / 5$ when 5 divides $x$. For $c=4,(10)$ has obvious solution $(x, y)=(30,5)$. The general solution $\left(x_{n}, y_{n}\right)$ is then obtained solving the unitary Pell equation $x^{2}-20 y^{2}=1$ whose particular solution $(x, y)=(9,2)$ acts as a generator: $x_{n}=30 r_{n} \pm 100 s_{n}$ and $y_{n}=30 s_{n} \pm 5 r_{n}$ with

$$
\begin{aligned}
& r_{n}=\left[(9+4 \sqrt{5})^{n}+(9-4 \sqrt{5})^{n}\right] / 2, \\
& s_{n}=\left[(9+4 \sqrt{5})^{n}-(9-4 \sqrt{5})^{n}\right] /(4 \sqrt{5}) .
\end{aligned}
$$

To $(x, y)=(30,5)$ is associated the solution $(a, b)=(6,5)$ of the original equation (9), defining the (simple) closed geodesic passing through $e_{0}=5 / 6$ of Kepler's case. Infinitely many closed geodesic with $\pi$-rational length are designed so (e.g., $e_{0}=20 / 21$ with $c=11$ - see Fig. 2 , etc.)

Remark. In Kepler's case, meridians are the shortest closed geodesics. Indeed, the length of a closed geodesic such that $\Delta \theta /(2 \pi)=p / q$ is $2 \pi q \sqrt{1-\left(1-\mu^{2}\right) e_{0}^{2}}$, not less than $2 \pi \mu q$. If closed geodesics shorter than meridians exist, it is necessarily for $q \leq 1 / \mu$. This implies $q=1$ or 2 in Kepler's case $(1 / \mu=\sqrt{5}<3)$, that is $e_{0}=0$ (meridians, precisely) for $q=p=1$, or $e_{0}=5 / 8$ for $q=2, p=1$, which has length $\pi \sqrt{11}>2 \pi$, since $p$ has to be chosen such that $1 \leq p \leq q$, $p \wedge q=1$.

\section{Convexity properties}

The meridian half-planes of $X$ are the subsets $\theta=$ cst. They are all isometric to $X_{0}=\{\theta=0\}$, and the metric $g$ has a flat restriction on them [3]: $g_{\mid X_{0}}=$ $\mathrm{d} r^{2}+\left(r^{2} / c^{2}\right) \mathrm{d} \varphi^{2}$, that is

$$
g_{\mid X_{0}}=\mathrm{d} r^{2}+r^{2} \mathrm{~d} \psi^{2}
$$




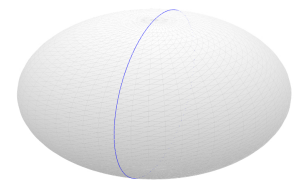

$e_{0}=0, L=2 \pi$

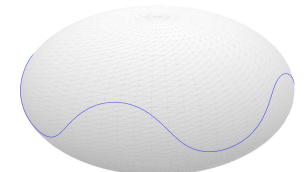

$e_{0}=15 / 16, L=\pi \sqrt{19}$

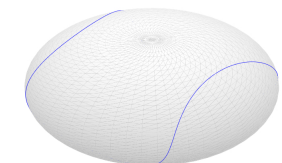

$e_{0}=5 / 8, L=\pi \sqrt{11}$

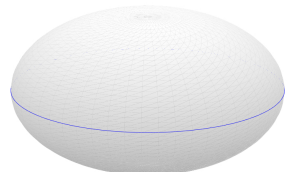

$e_{0}=1, L=2 \pi \sqrt{5}$

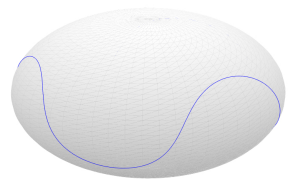

$e_{0}=5 / 6, L=4 \pi$

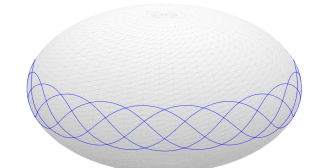

$e_{0}=20 / 21, L=22 \pi$

Figure 2: Closed geodesics in Kepler's case. The first five are the simple ones.

setting $\psi=\varphi / c, \varphi$ in ] $-\pi / 2, \pi / 2[$ (upper half-plane). The metric (11) is in polar form, and flat coordinates are $x=r \sin \psi, z=r \cos \psi$. This reduction is important in Kepler's case since such half-planes correspond to transfers towards circular orbits (where the transversality condition of Pontryagin maximum principle reads $p_{\theta}=0$, that is $\theta=\mathrm{cst}$ ). This is used in practice to initialize the computation of transfers towards the geostationary orbit.

The effect of the singularity $r=0$ is clear in this context.

Proposition 8. The manifold $(X, g)$ is not complete.

Proof. The separatrices $\varphi=$ cst, that is $\psi=$ cst in polar coordinates on $X_{0}$, define geodesics on $X$ which reach the singularity $r=0$ in finite time.

Regarding the weaker property of (geodesic) convexity, the following holds.

Proposition 9. A necessary condition for convexity of the manifold $(X, g)$ is $c \mu>1$.

Proof. The diameter of $\left(\mathbf{S}^{2}, g_{2}\right)$ is the half-length of the longuest simple closed geodesic which is clearly the equator: $\operatorname{diam}\left(\mathbf{S}^{2}\right)=\pi / \mu$. According to (8), the time $\sigma$ on the sphere is bounded over by $c \pi$. The supremum not being reached, the condition is necessary (no geodesic in $X$ can reach a point that projects onto a point of $\mathbf{S}^{2}$ further than $c \pi$ ).

Remark. Given two points on $X$ whose projections on the sphere are closer than $c \pi$ from each other, Lemma 4 combined with Propositions 11, 12 of 4 ensures the existence of a geodesic between them without conjugate point or point on the separating line. The sphere is indeed compact, so complete, and one just has to lift a minimizing geodesic onto $X$ thanks to the aforementioned lemma. This is not sufficient however to guarantee global optimality of the lifted geodesic since cut points not of the two previous kinds may exist because of incompleteness of the metric on $X$. It should moreover be noted that the two necessary conditions on global optimality (Theorem 1) and convexity are incompatible. In the limit flat case $c=\mu=1, X \simeq \mathbf{R}^{3}-\{0\}$ which is clearly not convex. 
Corollary 4. The metric is not geodesically convex on $X$ in Kepler's case $(c \mu=\sqrt{2} / 5 \leq 1)$.

The non-convexity is well depicted in meridian half-planes. In flat coordinates indeed, it is obvious that a geodesic with initial angle $\psi_{0}$ cannot reach a point with angle beyond $\pi+\psi_{0}$ because of the singularity $r=0$ when $c<1$ (see Fig. 4). Such a pair of points actually projects onto points on $\mathbf{S}^{2}$ further than $c \pi$, and the analysis in $X_{0}$ is sufficient as is now stated.

While optimality properties are related to infinitesimal or global injectivity of the exponential mapping, existence is connected to the presently discussed convexity issues, that is to surjectivity of the exponential. The geodesic flow on the Riemannian manifold $X$ is obtained through the exponential mapping, defined for small times on the whole cotangent space at the initial point, $\exp _{x_{0}, t}: T_{x_{0}}^{*} X \rightarrow X$, by

$$
\exp _{x_{0}, t}\left(p_{0}\right)=\Pi \circ \exp t \vec{H}\left(x_{0}, p_{0}\right),
$$

where $\exp t \vec{H}$ is the one-parameter subgroup generated by the Hamiltonian, and $\Pi: T^{*} X \rightarrow X$ the canonical projection. By homogeneity, we restrict ourselves to the level set $H=1 / 2$, parameterizing geodesics by arc length, or consider alternatively $\exp _{x_{0}}=\exp _{x_{0}, t=1}$ which, according to Hopf-Rinow, is only defined on an open subset of the cotangent bundle since the manifold is not complete. The same construction holds on the complete manifold $\mathbf{S}^{2}$, and for $y_{0}$ on the sphere, we set

$$
\Lambda_{t}=\exp t \overrightarrow{H_{2}}\left(\Lambda_{0}\right)
$$

where $\Lambda_{0}=T_{y_{0}}^{*} \mathbf{S}^{2} \cap\left\{H_{2}=1 / 2\right\} \simeq \mathbf{S}\left(T_{y_{0}}^{*} \mathbf{S}^{2}\right)$. Both $\Lambda_{0}$ and $\Lambda_{t}$ are Legendrian submanifolds of the fibered space of oriented contact elements [1] which is homeomorphic to the spherical cotangent bundle, $\mathbf{S}\left(T^{*} \mathbf{S}^{2}\right)$. The wavefront at time $t$ is the projection $W\left(y_{0}, t\right)=\Pi\left(\Lambda_{t}\right)$. Its singularities run along the caustic of the Lagrangian submanifold $L=\exp _{y_{0}} t \overrightarrow{H_{2}}\left(T_{y_{0}}^{*} \mathbf{S}^{2}\right)$, that is the set of singular values of $\Pi$ restricted to $L$.

According to Proposition 9, the surjectivity default of $\exp _{x_{0}}$ is described by the wavefront $W\left(y_{0}, c \pi\right)$, where $y_{0}$ is the projection of $x_{0}$ on $\mathbf{S}^{2}$ (see Fig. 3). The following lemma allows us to reduce the study to meridian half-planes.

Lemma 3. The longuest geodesic from any point on $\mathbf{S}^{2}$ to the equator is the negative half-meridian, $p_{\varphi}=-1$.

Proof. The quadrature (5) tells us that, for nonnegative $p_{\varphi_{0}}$, the equator is reached at time $t=t_{1}+T / 4$. By symmetry, it takes $t=-t_{1}+T / 4$ to reach the equator on a geodesic such that $-1<p_{\varphi_{0}}<0\left(-2 t_{1}>0\right.$ translation). This time increases towards $\pi / 2+\varphi_{0}$ which is precisely the time required to reach $\varphi=\pi / 2$ on the negative half-meridian defined on $H_{2}=1 / 2$ by $p_{\varphi_{0}}=-1$.

The picture in meridian half-planes thus characterizes surjectivity, as in Fig. 4 .

Proposition 10. Given $x_{0}$ in $X$, the exponential mapping $\exp _{x_{0}}$ is surjective if and only if $\varphi_{0}<\pi(c-1 / 2)$. 

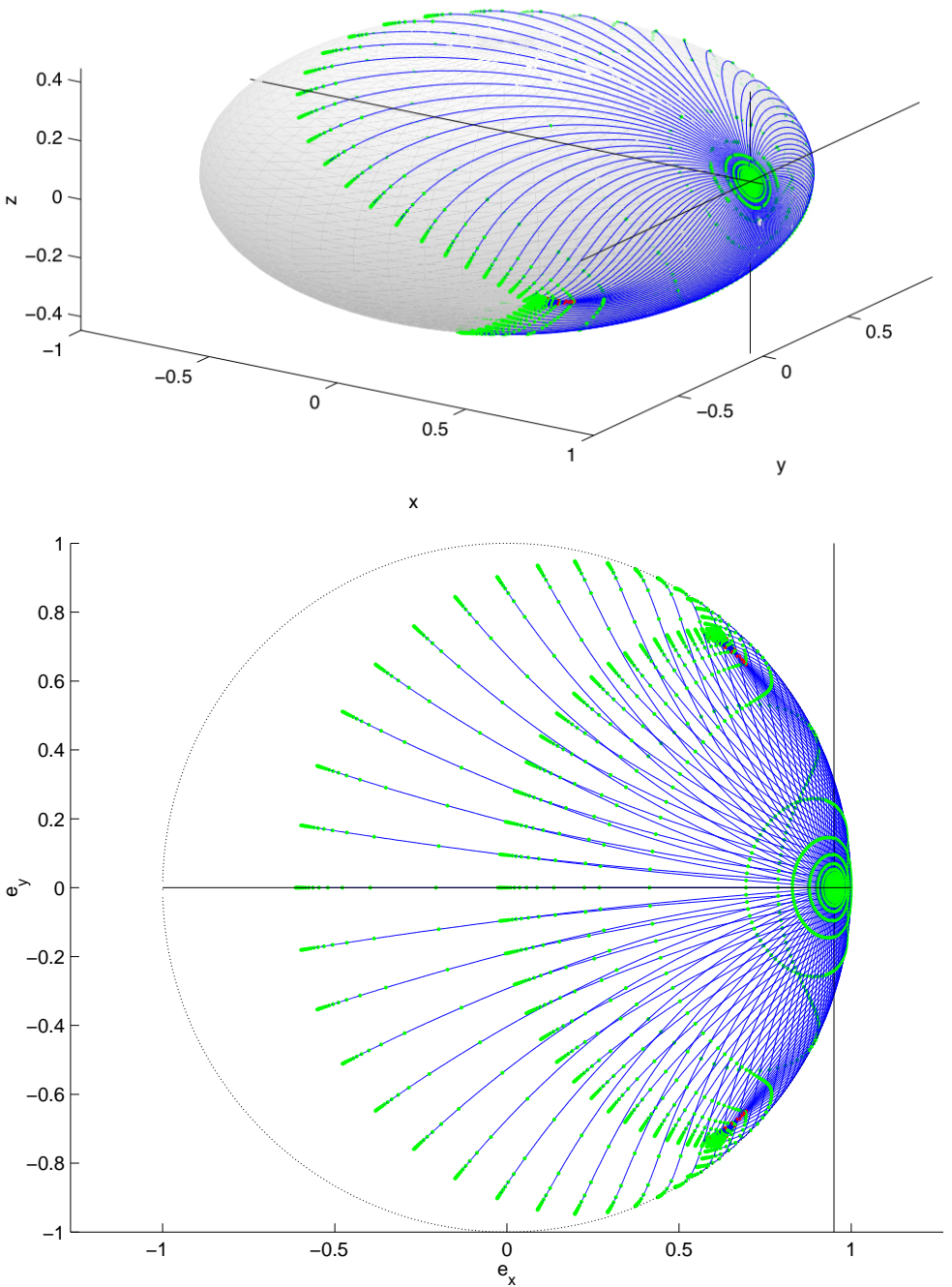

Figure 3: Above, wavefront on the sphere for initial condition $e_{0}=9.5 e-1$ and time $t=c \pi$ in Kepler's case $(c=\sqrt{2 / 5})$. The exponential mapping is surjective if and only $e_{0}<\arcsin (\pi(c-1 / 2)) \simeq 4.29 e-1$. Below, projection of this wavefront on the Poincaré disc, $\mathbf{D}$. The apparent contour describes the default of surjectivity in the space of ellipses, $X_{e}=\mathbf{R}_{+}^{*} \times \mathbf{D}$, that is before the analytic prolongation to $X=\mathbf{R}_{+}^{*} \times \mathbf{S}^{2}$.

\section{Optimality results}

The cut point on a geodesic is the first point where the geodesic ceases to be minimizing, and the first conjugate point is the point where moreover local optimality with respect to neighbouring trajectories is lost [12]. The conjugate locus is the set of first conjugate points on geodesics issuing from one given point. Ja- 


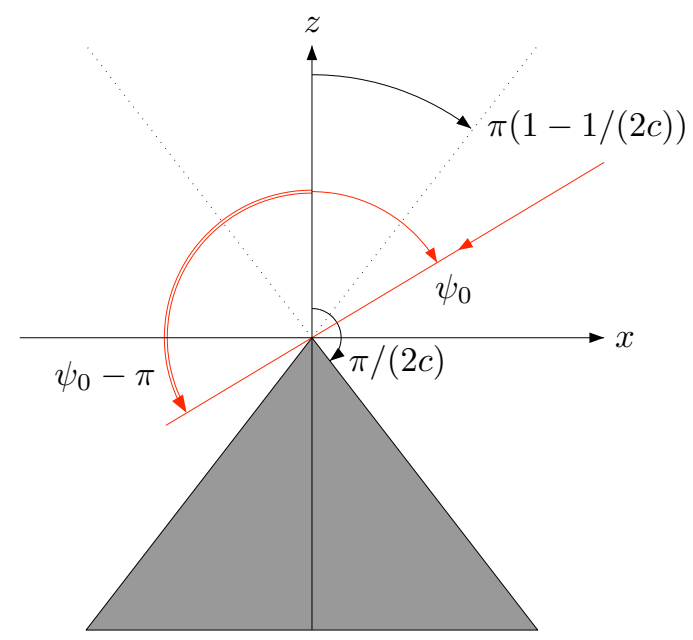

Figure 4: Geodesics in meridian half-planes issuing from a point such that $\psi_{0} \geq \pi(1-1 /(2 c))$ - that is $\varphi_{0} \geq \pi(c-1 / 2)$ - cannot reach points in the sector defined by $\psi \leq \psi_{0}-\pi$, and surjectivity is lost.

cobi's theorem, which extends to the more general framework of optimal control, asserts that the conjugate locus is a subset of the caustic, subset formed by the first singular values of the exponential mapping. On a complete Riemannian manifold, cut points are either conjugate points, or points on the separating line where two minimizing geodesics intersect 8 . We are thus conducted to examine immersivity and injectivity properties of the exponential mapping. To this end, we begin with a preliminary lifting lemma.

Lemma 4. Through two points on $X$ which project on $\mathbf{S}^{2}$ onto points whose distance is less than $\mathrm{c} \pi$ passes one geodesic.

Proof. If the two points project onto the same one on the sphere, they lie on a separatrix in some meridian half-plane, and the result is trivial. Otherwise, let $\sigma$ denote the distance between them, $0<\sigma<c \pi$. On the compact manifold $\left(\mathbf{S}^{2}, g_{2}\right)$, there is a (minimizing) geodesic of such length joining these points, which is lifted to a (non necessarily minimizing) geodesic in $X$ provided the following system admits at least one solution $\left(p_{r_{0}}, t\right)$ in $]-1,1\left[\times \mathbf{R}_{+}^{*}\right.$ (see lemma 2 and (7)),

$$
\begin{aligned}
p_{r_{0}} & =\left(\left(r_{2}^{2}-r_{1}^{2}\right)-t^{2}\right) /\left(2 r_{1} t\right), \\
t & =r_{1} \sin (\sigma / c) / \cos \left(\arcsin \left(p_{r_{0}}\right)+\sigma / c\right),
\end{aligned}
$$

where $r_{1}$ and $r_{2}$ are the $r$-coordinates of the two points on $X$. The (possibly degenerate) hyperbola (12) always intersects the second curve in the prescribed domain. Indeed, at $t=r_{1}$, the point $\left(r_{1},-1\right)$ belongs to the second curve and is below $p_{r_{0}}\left(t=r_{1}\right)=\left(r_{2}^{2}-2 r_{1}^{2}\right) /\left(2 r_{1}^{2}\right)>-1$. Conversely, when $t \rightarrow \infty, p_{r_{0}} \rightarrow$ $-\infty$ for the first curve whereas the second one has an horizontal asymptote defined by $p_{r_{0}}=\cos (\sigma / c)$. The two curves must therefore cross somewhere in ]$-1,1\left[\times \mathbf{R}_{+}^{*}\right.$.

Given $x_{0}$ in $X$, we denote $\operatorname{Cut}\left(x_{0}\right), C\left(x_{0}\right)$ and $L\left(x_{0}\right)$, the cut locus, conjugate locus and separating line of $x_{0}$, respectively. The same notation is used for $y_{0}$ 
in $\mathbf{S}^{2}$, and we also denote $B_{o}\left(y_{0}, \sigma\right)$ the open metric ball of center $y_{0}$ and radius $\sigma$ which is the union of wavefronts from $y_{0}$ and times less than $\sigma$. Let $x_{0}$ be a fixed point in $X$, and $y_{0}$ its projection on the sphere.

Proposition 11. A point belongs to the conjugate locus $C\left(x_{0}\right)$ if and only if it projects onto a point in $C\left(y_{0}\right) \cap B_{o}\left(y_{0}, c \pi\right)$.

Proof. If $x$ belongs to $C\left(x_{0}\right), x$ cannot be on a separatrix in a meridian half-plane starting from $x_{0}$ since a direct Jacobi field computation proves that separatrices are without conjugate points. As a result, $x$ projects onto a point $y$ of $\mathbf{S}^{2}$ at distance $0<\sigma<c \pi$ from $y_{0}$. Now, the exponential on $X$ is obtained from the one on the sphere. Namely,

$$
\exp _{x_{0}, t}\left(p_{0}\right)=\left(r\left(t, r_{0}, p_{r_{0}}\right), \exp _{y_{0}, \sigma\left(t, r_{0}, p_{r_{0}}\right)}\left(\left(p_{\theta_{0}}, p_{\varphi_{0}}\right) / \sqrt{2 H_{2}}\right)\right)
$$

where $\sqrt{2 H_{2}}=\left(r_{0} / c\right) \sqrt{1-p_{r}^{2}}$. A simple rank computation shows that, for positive $t$ and $\sigma=\sigma\left(t, r_{0}, p_{r_{0}}\right)$, $\exp _{x_{0}, t}$ is singular if and only if $\exp _{y_{0}, \sigma}$ is singular, too. Thus, $y$ is a conjugate point, and even the first one on the corresponding geodesic (there would be a conjugate point before $x$, otherwise). Conversely, if $y$ belongs to $C\left(y_{0}\right) \cap B_{o}\left(y_{0}, c \pi\right)$, there is a geodesic of length $0<\sigma<c \pi$ joining $y_{0}$ and $y$ on which $y$ is the first conjugate point. This geodesic is lifted to a geodesic in $X$ between $x_{0}$ and $x$ thanks to Lemma 4 . The previous rank computation entails that $x$ is a conjugate point of this geodesic, and that there cannot be conjugate points before since this would contradict the fact that $y$ belongs to the conjugate locus.

Proposition 12. A point in the separating line $L\left(x_{0}\right)$ projects onto a point in $L\left(y_{0}\right) \cap B_{o}\left(y_{0}, c \pi\right)$.

Proof. If $x$ belongs to $L\left(x_{0}\right)$, there are two minimizing geodesics issuing from $x_{0}$, $\gamma_{1}$ and $\gamma_{2}$, intersecting at a positive time $\bar{t}$. Then $r_{1}(\bar{t})=r_{2}(\bar{t})$, which implies $r_{1} \equiv r_{2}$ by virtue of Lemma 2 (parabolas of same curvature intersecting at two distinct points are identical). The two geodesics share the same $p_{r_{0}}$ and project so onto geodesics of same length on the sphere, $0<\sigma\left(\bar{t}, r_{0}, p_{r_{0}}\right)<c \pi$. These two geodesics are necessarily minimizing up to the projection $y$ in $B_{o}\left(y_{0}, c \pi\right)$ of $x$ on $\mathbf{S}^{2}$. There would be otherwise a cut point before $y$, say, on the projection of $\gamma_{1}$. Since $\mathbf{S}^{2}$ endowed with the metric $g_{2}$ is complete, such a point would be either a conjugate point, or a point on the separating line of $y_{0}$. In the first case, Proposition 11 implies that $y^{\prime}$ would be lifted to a conjugate point in $X$ along $\gamma_{1}$, prior to $x$, thus contradicting optimality of the geodesic up to $x$. In the second case, there would be a third minimizing geodesic starting from $y_{0}$ on the sphere, intersecting the projection of $\gamma_{1}$ at $y^{\prime}$, lengths being the same at the intersection. This new geodesic would clearly be lifted to a geodesic in $X$ intersecting $\gamma_{1}$ strictly before $x$, lengths being again the same, contradicting optimality of $\gamma_{1}$ anew.

On $\left(\mathbf{S}^{2}, g_{2}\right)$, cut loci are obtained as the closure of separating lines. Since the metric is analytic, the cut locus of any point is a finite tree whose extremities are singularities of the conjugate locus [11, 15, 17. We now give a complete description of these sets which turn to be completely similar to the cut loci on an oblate ellipsoid of revolution (see \$1, Proposition 2). 
Proposition 13. The cut locus of point of eccentricity $e_{0}=\sin \varphi_{0}$ on the sphere is the line $\left[\theta_{l}, 2 \pi-\theta_{l}\right]$, with $\theta_{l}=\pi\left(1-\left(1-\mu^{2}\right) e_{0}\right)$, included in the antipodal parallel of the point, $\varphi_{l}=\pi-\varphi_{0}$. The distance from the point to its cut locus is $t_{l}=\pi \sqrt{1-\left(1-\mu^{2}\right) e_{0}^{2}}$, and is reached at the cut point on the corresponding pseudo-equator.

Proof. In accordance with discrete symmetries discussed in $\$ 2$ intersecting geodesics of same length are obtained using $s_{1}$ or $s_{2}$. Clearly, intersections generated by $s_{2}$ come prior to those generated by $s_{1}$ (whose length is not less than $T / 2$, $T$ being the period). These intersections are located in the antipodal parallel, and a direct computation on the quadratures of Proposition 6] shows that the two geodesics defined on $H_{2}=1 / 2$ by a fixed $p_{\theta}$ and $\pm p_{\varphi_{0}}$ intersect at $t_{l}=$ $T / 2=\pi / \sqrt{1+\left(1-\mu^{2}\right) p_{\theta}^{2}}$. To conclude, it is sufficient to check that these two geodesics actually do not intersect before. This is obvious since the second one is obtained by a $-2 t_{1}$ translation of the first, with $-2 t_{1} \leq T / 2$, and since $\varphi$ is monotonic on the half-period $\left[t_{1}, t_{1}+T / 2\right]$ for nonnegative $p_{\varphi_{0}}$. The closure of the separating line is obtained letting $p_{\theta}^{2}$ tend to $G\left(\varphi_{0}\right)$ (letting $p_{\theta}$ tend to 0 , one gets for $t_{l}=\pi$ the intersection of half-meridians oppositely orientated located at $\theta=\pi$ which defines the center of the locus), that is for the associated pseudo-equator, so that $t_{l}=\pi \sqrt{1-\left(1-\mu^{2}\right) e_{0}^{2}}, \theta_{l}=\pi\left(1-\left(1-\mu^{2}\right) e_{0}\right)$.

As a result, we retrieve again the estimation of the injectivity radius of Theorem 1. Indeed,

$$
i\left(\mathbf{S}^{2}\right)=\inf _{\varphi_{0}} \pi \sqrt{1-\left(1-\mu^{2}\right) \sin ^{2} \varphi_{0}}=\mu \pi
$$

reached on the equator where the cut point is a conjugate point. This is in fact the case for any initial point, as a consequence of the following description of the conjugate locus.

Proposition 14. Conjugate times on a geodesic issuing from $\varphi_{0}$ defined on $H_{2}=1 / 2$ by $0<p_{\theta}^{2}<G\left(\varphi_{0}\right)$ and positive $p_{\varphi_{0}}$ are solution to

$\left(\cos \varphi_{0} /\left(\sin ^{2} \varphi_{0}-b\right)^{1 / 2}-a^{3}(1-b)\left(1-\mu^{2}\right) t / 8\right) \sin \left(a\left(t-t_{1}\right)\right)-\cos \left(a\left(t-t_{1}\right)\right)=1$

with $a, b$ and $t_{1}$ functions of $p_{\theta}$ as defined by Proposition 6 . The distance from any point to its cut locus is attained by a conjugate point on the corresponding pseudo-equator.

Proof. The constant adjoint state $p_{\theta}$ parameterizes $\Lambda_{0} \simeq \mathbf{S}\left(T_{y_{0}}^{*} \mathbf{S}^{2}\right)$ whenever $p_{\varphi}$ is nonzero. A straightforward differentiation of the quadratures with respect to $p_{\theta}$ in such cases gives the result. Letting $p_{\theta}^{2}$ tend to $G\left(\varphi_{0}\right), \sqrt{14}$ degenerates in $\sin (a t)=0$ whose first admissible zero is $T / 2$, which is thus the cut and first conjugate point on the associated pseudo-equator.

An example of cut and conjugate loci is represented Fig. 5 in Kepler's case. The conjugate locus is a deformation of the locus obtained for the oblate ellipsoid of revolution to which $\left(\mathbf{S}^{2}, g_{2}\right)$ is conformal. Its astroidal structure (i.e.with four cusps, two equatorial and two meridional) is studied in [4 where sufficient conditions to have such a conjugate locus and a simple antipodal cut are given in terms of properties of the function $\Delta \theta$ defined by (6). 

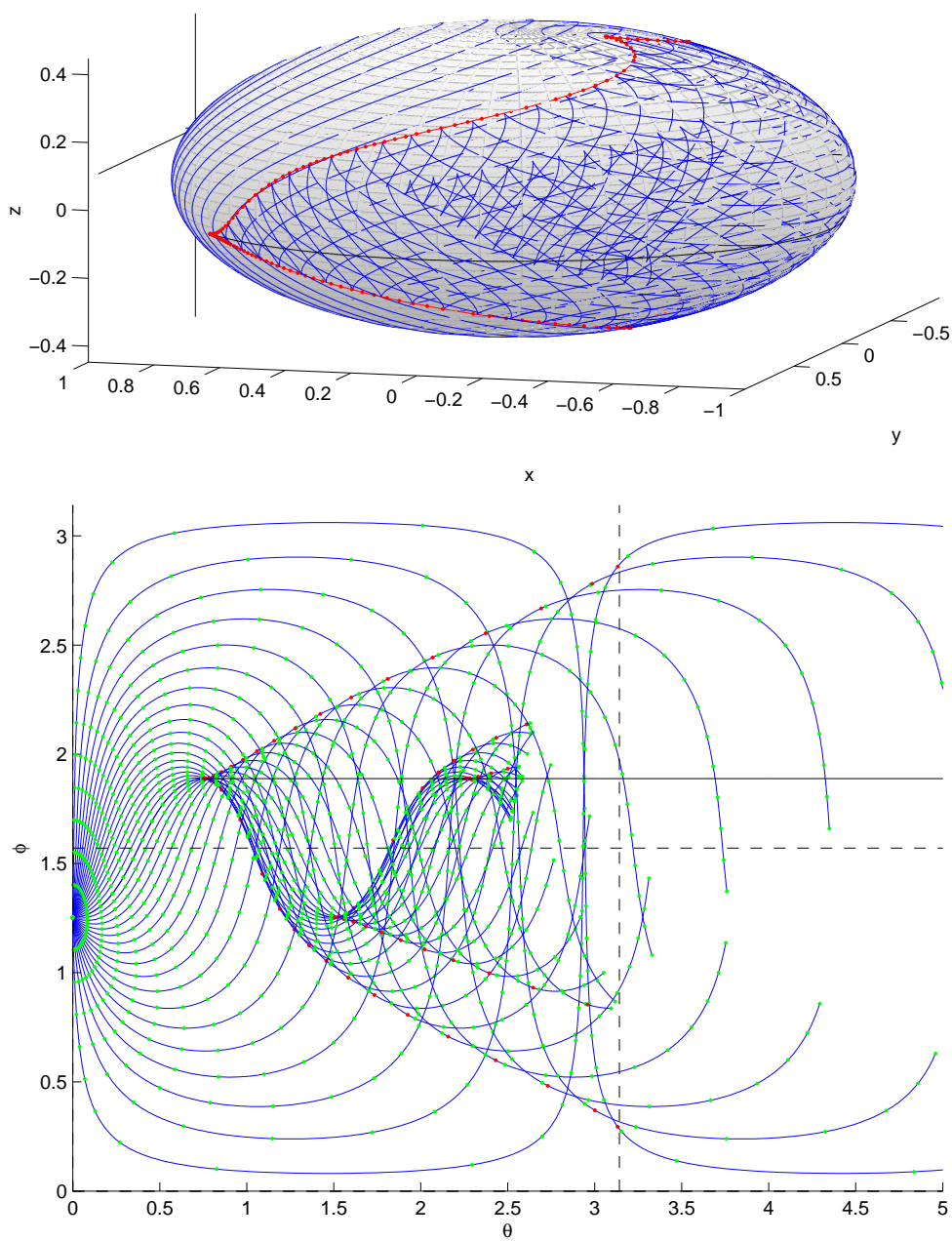

Figure 5: Wavefront, conjugate and cut loci for $e_{0}=9.5 e-1$. The bifurcation of the wavefront is observed on $\left(\mathbf{S}^{2}, g_{2}\right)$-realized as the oblate ellipsoid to which it is conformal-, and the swallowtail singularities appearing run along the caustic containing the conjugate locus. The inclusion of the cut locus in the antipodal parallel is clearly illustrated in $(\theta, \varphi)$ coordinates.

Corollary 5. A necessary and sufficient condition for a geodesic issuing from a point on $X$ with eccentricity $e_{0}$ not to have conjugate points or points on the separating line is $e_{0} \leq \sqrt{\left(1-c^{2}\right) /\left(1-\mu^{2}\right)}$. In Kepler's case, the condition is $\varphi_{0} \leq \pi / 3$.

Proof. Simply write that the distance from the projection of the point on $\mathbf{S}^{2}$ to its cut locus (see Proposition 13) is not less than $c \pi$.

We end the paper going back to the original non-extended averaged metric (1) on the space of ellipses, $X_{e}=\mathbf{R}_{+}^{*} \times \mathbf{D}$. The last result essentially asserts that Kepler's geodesics may only lose optimality because of completeness - that is existence-issues. 
Theorem 2. Conjugate loci and separating lines of the averaged Kepler metric on the space of ellipses are always empty.

Proof. According to the previous analysis, conjugate points or points on the separating line define cut points when projected to the sphere. Now, cut loci on $\mathbf{S}^{2}$ are included in antipodal parallels, so that cut points can only be reached by crossing the equator, that is the boundary $e=1$ of $X_{e}$. There are no such geodesics in the space of ellipses.

\section{References}

[1] V. I. Arnold, A. N. Varchenko and S.M. Gusein-Zade, Singularities of differentiable maps, Vol. 1, Birkhäuser, 1988.

[2] A. Bolsinov and A. Fomenko, Integrable geodesic flows on two-dimensional surfaces, Kluwer, 2000.

[3] B. Bonnard and J.-B. Caillau, Riemannian metric of the averaged energy minimization problem in orbital transfer with low thrust, Ann. Inst. H. Poincaré Anal. Non Linéaire 24 (2007), no. 3, 395-411.

[4] B. Bonnard, J.-B. Caillau, R. Sinclair and M. Tanaka, Conjugate and cut loci of a two-sphere of revolution with application to optimal control, HAL preprint (2008), no. 00212075, 1-20 (url: hal. archives-ouvertes.fr/hal-00212075).

[5] B. Bonnard, J.-B. Caillau and E. Trélat, Geometric optimal control of elliptic Keplerian orbits, Disc. Contin. Dyn. Syst. Ser. B 5 (2005), no. 4, 929-956.

[6] D. E. Chang, D. F. Chichka and J. E. Marsden, Lyapunov-based transfer between Keplerian orbits, Disc. Contin. Dyn. Syst. Ser. B 2 (2002), no. 1, $57-67$.

[7] G. Darboux, Leçons sur la théorie générale des surfaces, Tome III, Gauthiers-Villars, 1914.

[8] M. P. do Carmo, Riemannian geometry, Birkhäuser, 1992.

[9] D. Elerath, An improved Topogonov comparison theorem for non-negatively curved manifolds, J. Differential Geom. 15 (1980), 187-216.

[10] R. Epenoy and S. Geffroy, Optimal low-thrust transfers with constraints: generalization of averaging techniques, Acta Astronaut. 41 (1997), no. 3, $133-149$.

[11] H. Gluck and D. Singer, Scattering of geodesic fields I and II, Ann. of Math. 108 (1978), 347-372; and 110 (1979), 205-225.

[12] W. P. A. Klingenberg, Riemannian geometry, de Gruyter, 1982.

[13] W. P. A. Klingenberg, Poincaré's closed geodesic on a convex surface, Trans. Amer. Math. Soc. 356 (2004), 2545-2556. 
[14] H. von Mangoldt, Über die jenigen Punkte auf positiv gekrümmten Flächen, welche die Eigenschaft haben, daß die von ihnen ausgehenden geodätischen Linien nie aufhören, kürzeste Linien zu sein, J. Reine Angew. Math. 91 (1881), 23-53.

[15] S. B. Myers, Connections between geometry and topology I and II, Duke Math. J. 1 (1935), 376-391; and 2 (1936), 95-102.

[16] T. Nagell, Introduction to number theory, Wiley, 1951.

[17] H. Poincaré, Sur les lignes géodésiques des surfaces convexes, Trans. Amer. Math. Soc. 5 (1905), 237-274.

[18] M. Tanaka, On the cut loci of a von Mangoldt's surface of revolution, J. Math. Soc. Japan 44 (1992), 631-641. 